\title{
Reference Face Based Technique for Unconstrained Face Recognition from Images Gallery
}

\author{
Ranbeer Tyagi ${ }^{1}$, Geetam Singh Tomar $^{2 *}$, Laxmi Shrivastava ${ }^{3}$ \\ ${ }^{1}$ Deptt. of E\&TC, MITS, Gwalior \& Uttarakhand Technical University, Dehradun, \\ India \\ ${ }^{2}$ Deptt. of Electronics \& Communication, THDC-IHET Tehri Garhwal Uttarakhand, \\ India \\ ${ }^{3}$ Deptt. of Electronics Engineering, MITS, Gwalior (M.P.) India \\ ${ }^{1}$ ranbeertyagi85@gmail.com
}

\begin{abstract}
This paper represents the Id Quality of un-constrained face inside an unconstrained environment. It provides capabilities like discrepancy, illumination, and expression difference techniques. It might be useful for both retrieve image (Face) and identification (Recognition). At present plenty of way of top view face recognition can be found. In last few years, for Computer vision, numerous face recognition techniques have been organized. But, actualworld face detection demands a difficult works. The curiosity about unconstrained helpful face recognition keeps growing utilizing the detonation of online press for instance community methods, and video surveillance recording wherever experience analysis is of great importance. In this analysis, it is appeared to handle status inside the scenario of chart assumption. We're in a position to determine a magical experience employing a Varied Technique. This study lights out the choices suggested for unconstrained face recognition quality area and suggesting the solution to be utilized by Reference Face Based Technique (From Gallery Image) centered face recognition. RFG recognition is used in grouping with DCT locality sensitive hashing for efficient recovery to guarantee scalability. Objective of this research is Reference Face Based Technique focused on unconstrained face recognition to enhance the demonstration quality. The Simulation of proposed techniques will be completed through the use of MATLAB.
\end{abstract}

Keywords: Reference Face Based Technique, Unconstrained Face, DCT, RFG, Guide Block.

\section{Introduction}

Now days have been the great requirement for Image Fix and face recognition. The exceptionally review region has been face-recognition in Computer vision to get the last mix of years. Face recognition techniques in many cases are useful for protection places aside from are steadily more acquiring utilized in numerous uses. It might determine a complete business within a digital image by analyzing styles that is clearly a type of biometric software objective. For example, employs facial recognition to tell apart among people inside the Kinetic motion gaming system.

Article history:

Received (March 13, 2019), Review Result (May 26, 2019), Accepted (August 9, 2019) 
Now a day's the endemic planet, the necessity to corporeal possessions or to continue the protection of data is contrasting together steadily larger and steadily harder. By second-to minute it is focused on when it comes to the crimes of reputation permit scam, processor breaking's by hackers, or protection breaches within a business or administration workplace [12]. The region of "biometrics" is called this type of executive. Enveloped from the number of biometric acknowledgement practices, the physical methods (fingerprint, meeting, and genetics) are additional Continuous than systems in efficiency staff (keystroke, voice-print). Face recognition may be the unique of the several biometric methods to be able to obtain the faculties of together elevated accuracy and small intrusiveness. It is the accuracy of the bodily appears to with no being uncomfortable. Consequently of the, unconstrained face recognition has extended the notice of pupils in locations by protection, attitude, and digital image processing, to computer vision. Many computations have been organized intended for face recognition[17]. Whilst plan safety and dinner handle are it large thoroughly mentioned uses, face recognition in addition has confirmed helpful in preceding Disk in-series meting out places. The various applications of unconstrained face recognition are of the activities in a variety of contexts for instance checking verifying, community advertising, and personal-computer discussion. It constructed uncover of regularly unique or verifying a person starting an image or film models. Experience confirmation or experience acceptance will be the difficulty of arrived at a choice whether known match of images is of the equivalent person or not[18].

\subsection{Practical uses and its Challenges:}

Unconstrained face recognition is of more helpful use that will be nonetheless difficult due to the next components: Present variance, Imbalance, Lighting variance, Phrase variance, Standard blurs. That is used towards the accuracy of face recognition methods depreciates quickly in unconstrained locations[11], [19][20][21][22]. This could be recognized to degradations creating by cloud, change in illumination, present, and expression, unfinished occlusions etc.

For encounter identification, we see the texture and form parameters of the product which might be separated from imaging guidelines, for example pose and illumination [26]. There are two methods of capture:

Video imaging: Video imaging[25]uses video cameras. System functionality depends on exact location, angle of the head and nearby illumination situation.

Thermal imaging: Thermal imaging along with facial temperature variation has more accuracy $\&$ it is very costly.

\subsection{COMPONENTS OF FRT}

All recognition and authentication technologies function using the following:

Capture: A behavior or Bodily test is captured by the machine throughout registration as well as in confirmation or identification procedure.

Extraction: Unique data is extracted from the sample.

Comparison: Compared with a new sample.

Match/ non match: The program determines if the features extracted from your newest samples are an equivalent or maybe not. It starts using a picture, attempting to locate a 
individual in the image. Spot the skull and eye position. A medium is then residential based on the distinctiveness of the expression that was exacting [3].

Implementation of face Recognition System following Stages: Data acquisition, Input processing, Face image classification and decision making.

Data Acquisition: The input can be recorded as video. A sample of 1 second duration consists of 25 body video sequence. More than one digicam can be used to make a 3D rendering. It can help to shield against the use of photographs to unauthorized access[15][16].

Input Processing: A preprocessing element also takes care of the lighting that is encircling and marks the vision spot state and color variance. Following the face is recognition; localization and normalization are carried away. The look of the face may change considerably all through speech and due to facial expressions [4].

How Face Recognition System works: Face recognition system performs by applications that are certain. There are about 80 nodal points on a human face. Here are the few nodal points which might be quantified by the applications: Space between the eyes, Size of the nose, Degree of the eye plug, Cheek Bones, Jaw-line and Chin. These factors are quantified to create a numeric signal, a string of amounts that represent a face in the $\mathrm{db}$ [1][2]. This code is called face print. Simply 14 to 22 nodal points are essential for discovering face and finished the acknowledgment process. Nodal Point, Alignment, Normalization, Representation and Matching.

Nodal Point: Any of two positions on the axis of a lens or added visual association, resolute by increasing an incident oblique beam and the equal refracted beam to the axis designed for the pair of beams that is parallel external the visual format [5].

Alignment: Once a face is detected the system determines pose, dimensions and the heads posture. A face has to be switched at least 35 degree toward the cam for the machine to join up it.

Normalization: It is performed regardless of the heads place and distance from the cam.

Representation: This code procedure license for easier comparison of the just grabbed facial data to preserved.

Matching: The new recorded face data is when compared with the data that is preserved.

\section{Related work}

Mainly, current facial recognition methods function by numeric crucial guidelines called experience pictures. Such methods determine 80 nodal facets about the individual experience. In this situation, nodal facets are end points used to determine flexible of the person's experience, for example the length or width of the nostril, the profundity of the interest plugs as well as the consideration of the nerve fillets. The cosmetic skin printing might afterwards be used such as a foundation for distinction with info jailed by activities in an impression or movie. The dissimilarity flanked by face detection and reputation may be the proven fact that in acknowledgement. Face-recognition continues to be determined expansively; about the otherhand, real-planet face-recognition quiet locations a challenging objective. Their state useful for unconstrained wise face recognition keeps growing through the growth of online press for 
instance community techniques, and film assertion conserving everywhere experience analysis is of essential importance.

In [6] authors Face-recognition has been assessed thoroughly; nevertheless, real World face recognition yet stays an occupation that is difficult. The curiosity in unconstrained useful Facerecognition is increasing utilizing the upsurge of online media and, for example internet sites Video surveillance-video wherever experience assessment is of value that is substantial. It is determine a different experience having an outside RFG. A RFG is created and reputation of the given experience is achieved by analyzing it towards the activities inside the constructed RFG.

$\operatorname{In}[7][8]$ authors give a broad review on unconstrained face recognition and its application. The authors elaborate different method and comparison used in facial feature recognition and extraction. In [13] authors discussed colour to grey image scale of an image using different techniques and compare the performance. In [14] authors compare the performance of Canny and Sobel filters used for face recognition. The authors concluded that the Canny filters better performance.

A RFG is done and confirmation of the acknowledged experience is achieved by contrasting it towards the activities inside the constructed RFG[23][24]. Centrality methods are employing to recognize individual activities inside the RFG. The determination useful for having such places is mounted on the conventional discrepancy of experience images after face detection, in calculation to utilizing a dimension-free method with no need for aesthetic feature positioning detection (e.g., eyes). Many specific-of the-sculpture face recognition computations need mixed activities to trigger through elevated accuracy.

A RFG is identification and produced of the given by evaluating it to the faces inside the made RFG experience is accomplished. Measures are employed to establish special encounters in the research experience chart. As multimedia's explosion has been seen in the past decade, the multimedia data's control and research, including films and photos, are of broad awareness. Among these data, experience pictures and films have a fraction that is significant [27]. The motivation for having areas relates to the common mis-alignment of experience images after face-detection, along with having a level- free approach without the need for cosmetic characteristic level recognition (e.g., eyes). Many state-of the- art face recognition algorithms involve aimed people to function with high accuracy. By using multiple parts with different level and translation setting it eliminate the importance of experience position.

\section{Major Contribution of this work}

The aim of study because of diversity of face recognition techniques have been organized significantly from the last several years, many of them have achieved motivating effectiveness, they simply perform nicely under precisely-managed conditions. In various real life purposes for smart surveillance methods in frantic locations, personal activities are very quickly occluded by additional substance such conditions that will be complicated to acquire complete substitute experience images for identification. Within the same interval as regular, alongside person, capabilities identifications in certain conditions may everlastingly be indefinable; there yet stays a massive space among computerized methods and person's effect on known faces. In addition to minimize this space, big annotated sets of imagery are favored utilizing the reasons that are envoy of the final outcome objectives of unconstrained face recognition. The reference face based technique provides better results for unconstrained face recognition with good matching the results. 


\section{FACE RECOGNITION}

It is an automatic face identification system which utilizes computer applications to identify faces from digital images or videos. It helps to verify and identify a face from a pool of digital faces in the data base. Face authentication is a process in which a pair of faces is matched to find whether the person being identified is same as in the pool of images or not.

Face recognition uses various algorithms to identify a person from video from of a source video stream. Typically, face identification is done by using selected facial features. These feature points of pair of images are matched to find a image with maximum matching which helps in the process of facial authentication. It is widely used in security system e.g. video surveillance, biometrics [9] etc. It is also penetrating in the commercial and marketing field [10].

\section{CONSTRAINED FACE RECOGNITION}

Development in the field of constrained face recognition system leads to unconstrained face recognition. This process of development faced various challenges as unconstrained face recognition process incorporates variation in illumination, image resolution, background noise, facial expressions, and occlusion related problems (e.g. video surveillance).

Forensic investigation is one of the major applications of face recognition methods. And the fundamental difference between the present and tradition forensic investigation faces in the number of sources. It takes to identify and verify a person's face. Forensic investigations includes sources like video tracks, still images taken by other cameras (eg. Mobile phones), face designing models and verbal explanation by some related person. While, the traditional method of face matching verify a person's identify based on single source media (eg. Video track, video database etc.). Fig.1 shows the Sample of constrained face images.
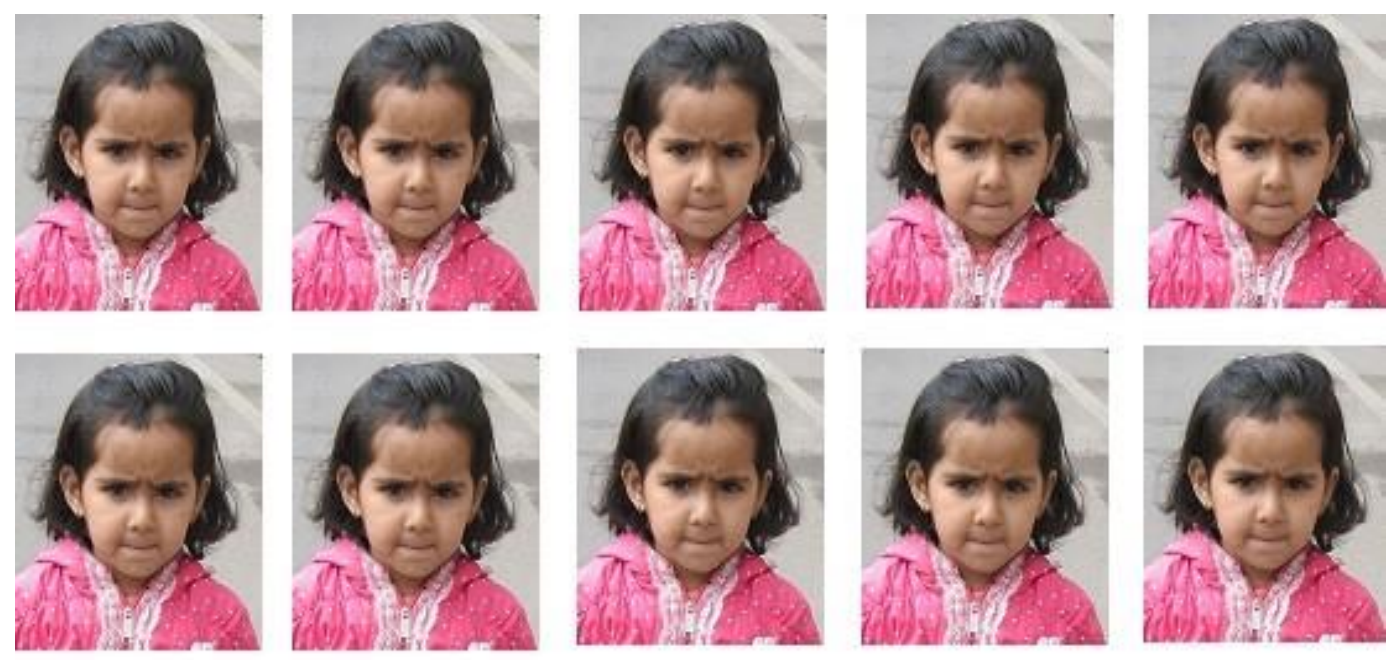

Figure. 1. Sample of constrained face images

\section{UNCONSTRAINED FACE RECOGNITION}

The unconstrained recognition problem has become more dominant due to varying nature of applications under dynamic environment. 
The unconstrained recognition problem has become more dominant due to varying nature of applications under dynamic environment. Video surveillance, forensic systems, and computer vision has propelled the research in unconstrained environment.

Face recognition under controlled environment is a mature technological method as various algorithms have achieved almost error less recognition. Controlled face recognition has achieved a benchmark in the controlled recognition methods. And, it is dominantly used in biometric systems where subjects voluntarily pose themselves for the process of identification.

Video surveillance poses a big challenge for facial recognition as the subject is not voluntarily posing themselves which increases the false detection rate. Applications under uncontrolled conditions (e.g., varying illumination, expression, and pose, etc.) are known as uncontrolled face recognition. It is a more practical approach as it helps to recognize faces under real world varying environment. And, it is a challenge due to following factors:

Pose Variation: Any change in facial object due to change in direction of the subject (being recognized) with respect to the direction of camera.

Misalignment: When cropped faces are not aligned to get the most appropriate feature vector from the face, is called as Misalignment.

Illumination Variation: Any change in lighting condition affects the appearance of a face under recognition.

Expression Variation: Change in facial expression under different emotional phases or reactive phases pose a challenge in facial recognition.

Fig.2 illustrates the examples of unconstrained faces. It clearly shows that the appearance of a person varies significantly due to chance in any of above mentioned factors.

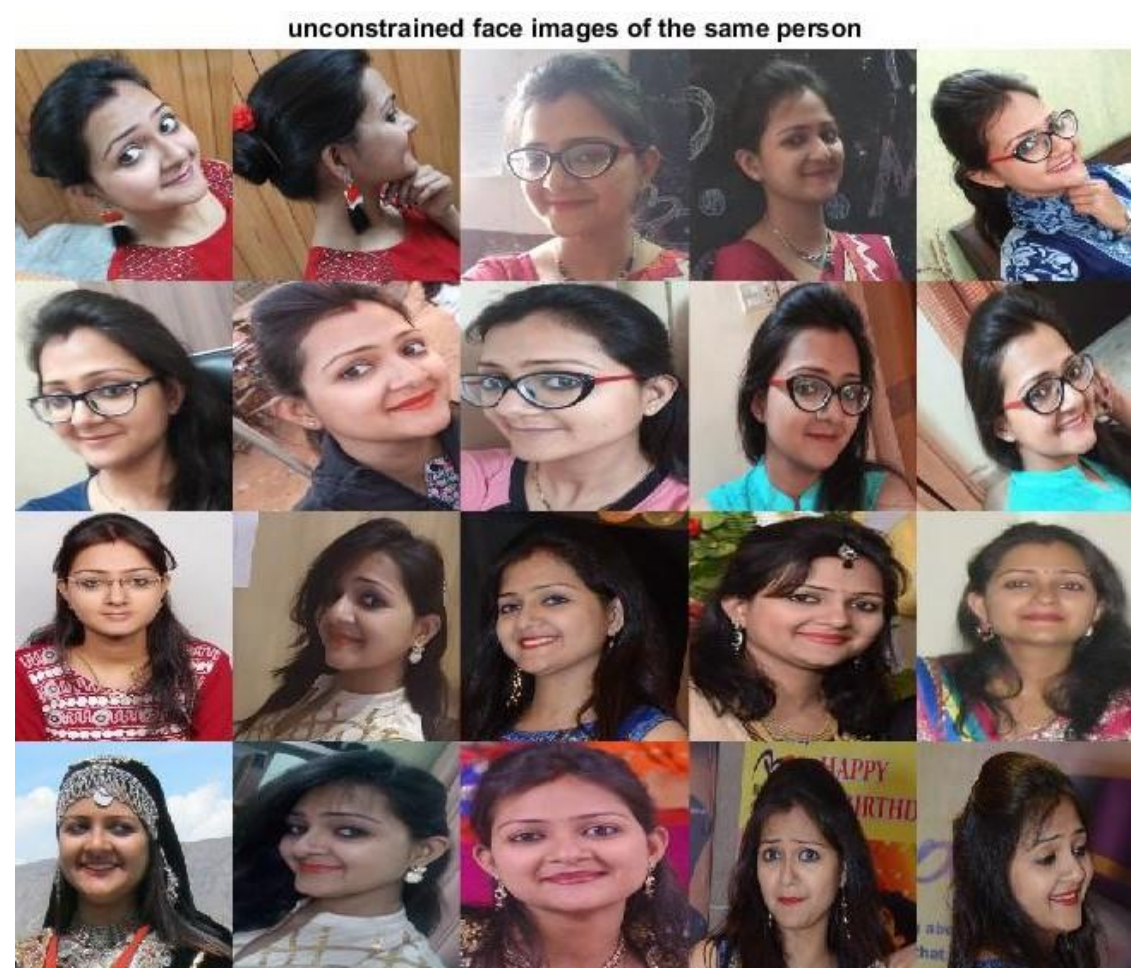

Figure. 2. Matrix representation of unconstrained face images of the same person under various environmental and physical constraints e.g. variations in pose, illumination, expression, and alignment. 


\section{CHARACTERISTIC EXCTRACTION}

Feature extraction and pattern recognition forms the base of Image Processing and Machine Learning respectively. And, both of these techniques start with an initial set of data (facial images in case of facial recognition). Then features are derived from the set of data which help to facilitate the recognition and learning process of the algorithm used to identify the face of a person. Feature extraction is related to dimensionality reduction problem.

A data is deemed to be redundant when it is too large to be processed. To process each data through an algorithm, the large data set has to be transformed into a reduced set of features (a.k.a. feature vector). Feature selection is a method to determine a subset of initial feature using which it is possible to identify the subject successfully.

\section{TECHNICAL APPROACH}

This work comprises two steps for face recognition. First, prepare the reference face graph and second find the descriptors of reference faces from the reference face graph. The reference face graph is an interconnection of nodes where each node represents a reference face and each reference face is obtained from multiple images and with their corresponding poses, experimental illumination. All the images corresponding to a reference face are called as reference basis set. A basis descriptor of an image is used to find the degree of similarity of the reference face with respect to the basis vectors. The RFG node centrality metric is used to find a reference face descriptor (RFD). Figure 3 illustrates the system diagram for RFG based recognition.

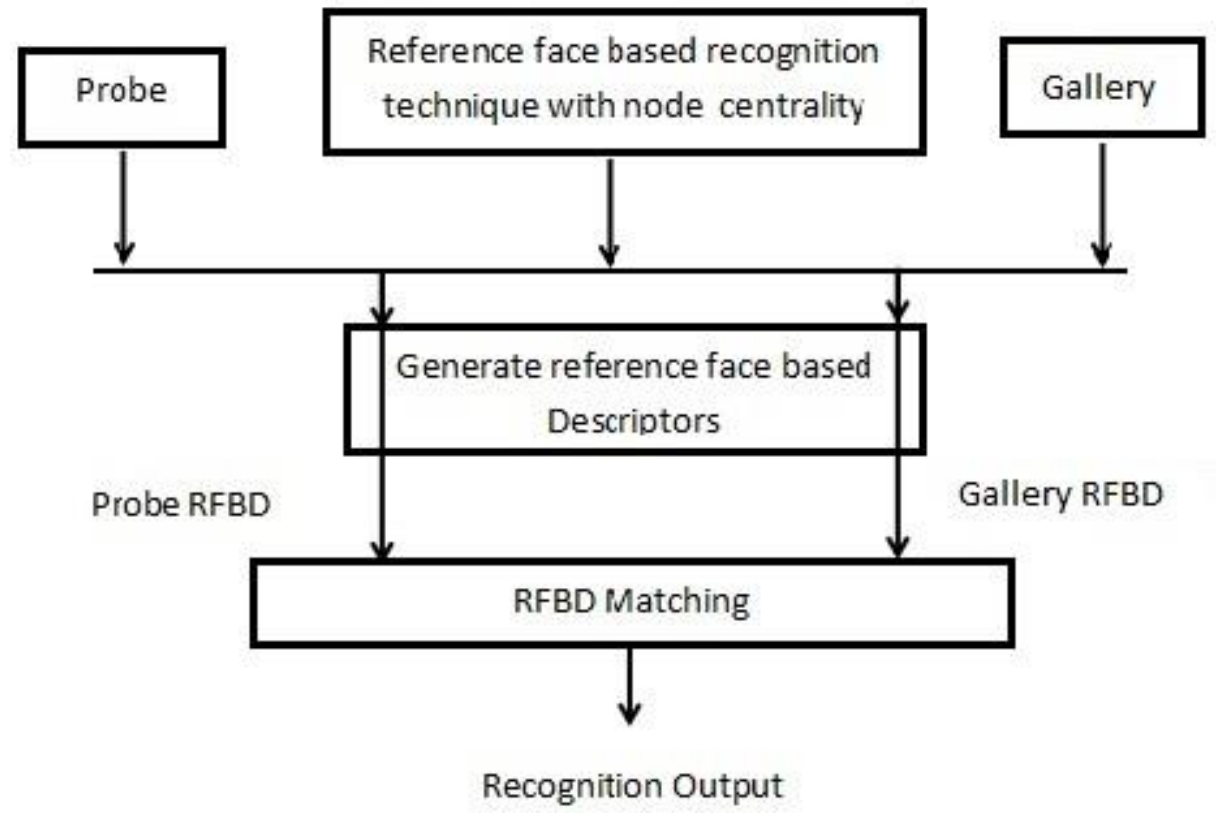

Figure 3. System Diagram for Reference Face Based Recognition Technique.

\section{BUILDING THE REFERENCE FACE BASED TECHNIQUE}

Reference Face Initialization: Initializing a reference face is a process which defines a reference face based structure by prepopulating it with a set of reference faces. A reference basis is obtained from the reference faces. Figure 4 represents a set of $\mathrm{N}$ reference faces. 
To further process the image, each image from the reference basis set is partitioned into regions. It uses eight partitioning methods to create 4, 9, 16, 25, 49, 64, 81, and 100 regions. These partitioned regions do not overlap with each other. In each method, the regions are retranslated. And, for each patch in a partitioning, a feature vector is extracted. Feature vectors of a person in the reference set help to construct a reference face Bi and DCT hash based similarity[6].

In this algorithm, need for face alignment is removed which is commonly used in state-ofart face recognition algorithms. Thus it is adaptable to work for nonaligned images with varying scales. Figure 7 depicts a sample partitioning for a reference basis set image.

A node $B_{1}, 1=1, \ldots, N$ is assigned to each reference face in the RFG. For a complete RFG, each $B_{1}$ is connected to all other nodes via a direct edge e. $K_{l t}$ represents the weight of edge between node 1 and node $t$. And, $V_{l t}$ shows the similarity between reference face $B_{l}$ and $B_{t}$, which is defined as:

$$
V_{l t}=\operatorname{sim}\left(B_{l}, B_{t}\right)=\max _{i, j} \operatorname{sim}\left(B_{l}^{i}, B_{t}^{j}\right)
$$

Where $B_{l}^{i}$ and $B_{t}^{j}$ refer to images $\mathrm{u}$ and $\mathrm{v}$ of reference faces $\mathrm{B}_{1}$ and $\mathrm{B}_{\mathrm{t}}$, respectively. Equation 1 represents the similarity between two reference faces It is used to get maximum similarity between images of two reference faces.

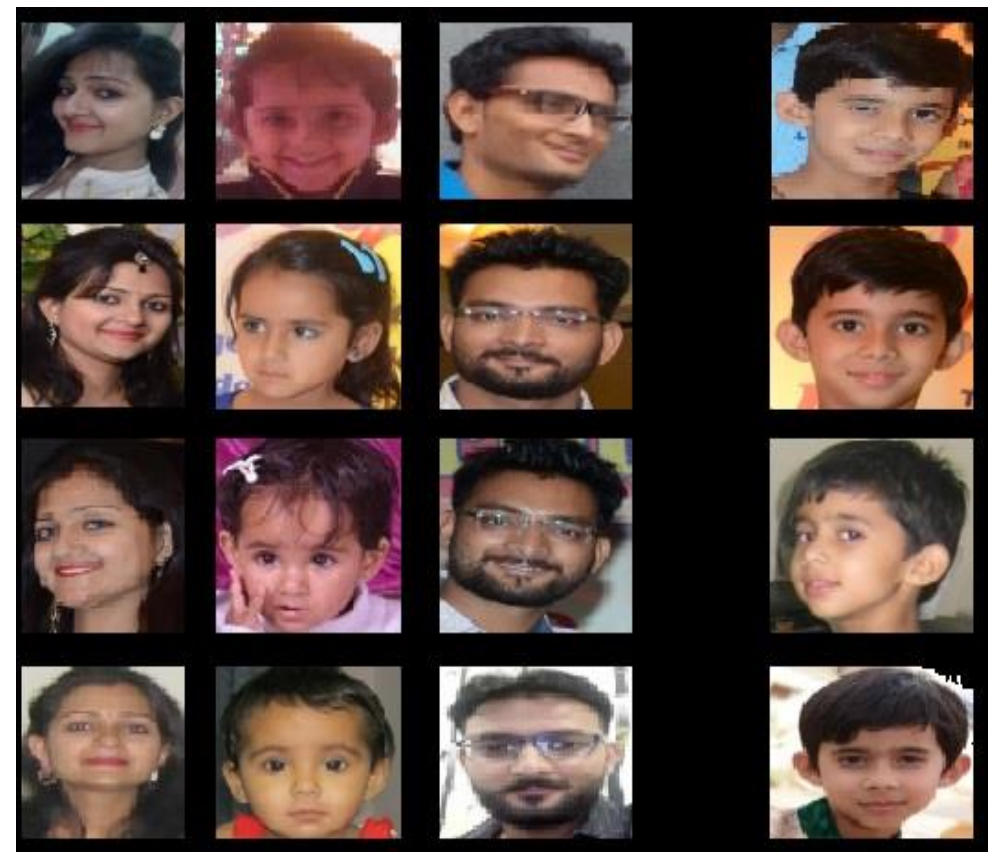

Figure. 4. Reference basis set: a set of images containing multiple individuals (reference faces). Each reference face has multiple images with various posesexpressions, and illumination settings
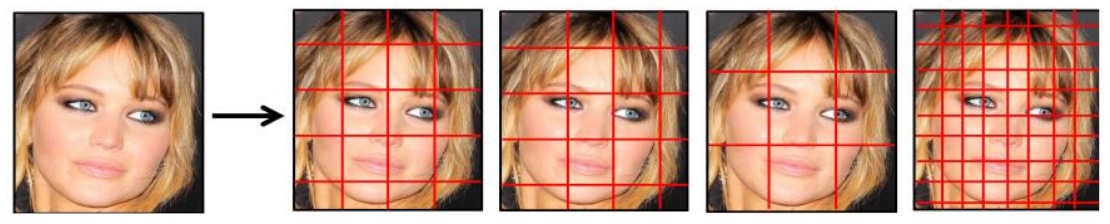

Figure. 5. An example of oversampling regions[6]. 
Node Centrality Measures: It is used to determine the more distinctive reference faces for a RFBT which is a static un-directional weighted graph with base $\mathrm{B}=(\mathrm{W}, \mathrm{F})$ consists of a set of nodes $\mathrm{W}$ and a set of edges $\mathrm{F}$. The linkage structure is used to propagate labels among various nodes. It measures the centrality of a reference face which suggests about the degree of importance of the reference face in the RFG. Three measures of node centrality are used to measure the centrality of each reference face including degree, between-ness, and closeness for weighted graphs [6].

Generally,for a given weighted graph, degree of a node is extended to the sum of weights and named as node strength $\mathrm{h}$. B is an in-directed weighted graph,

so it is preferred to use node strength instead of degree as a measure of centrality.

\section{REFERENCE FACE BASED TECHNIQUE}

In order to check the effectiveness of current work, a through face verification and identification using the RF technique is performed, Steps of which are mentioned below:-

- Verification: it is a matching process which compares the current face images with the existing data base (pair matching). In order to obtained the threshold required for proper verification a linear SVM classifier trained on images which were not used during evaluation.

- Identification: This step probes a facial image from the gallery also known as data base resulting in a set of facial images ranked by similarity.

The proposed RF based method is compared under various metric with several state-of-theart algorithms on multiple face databases. RF recognition does not require any face alignment. The detected faces with different algorithms have different resolutions depending on the original image size.

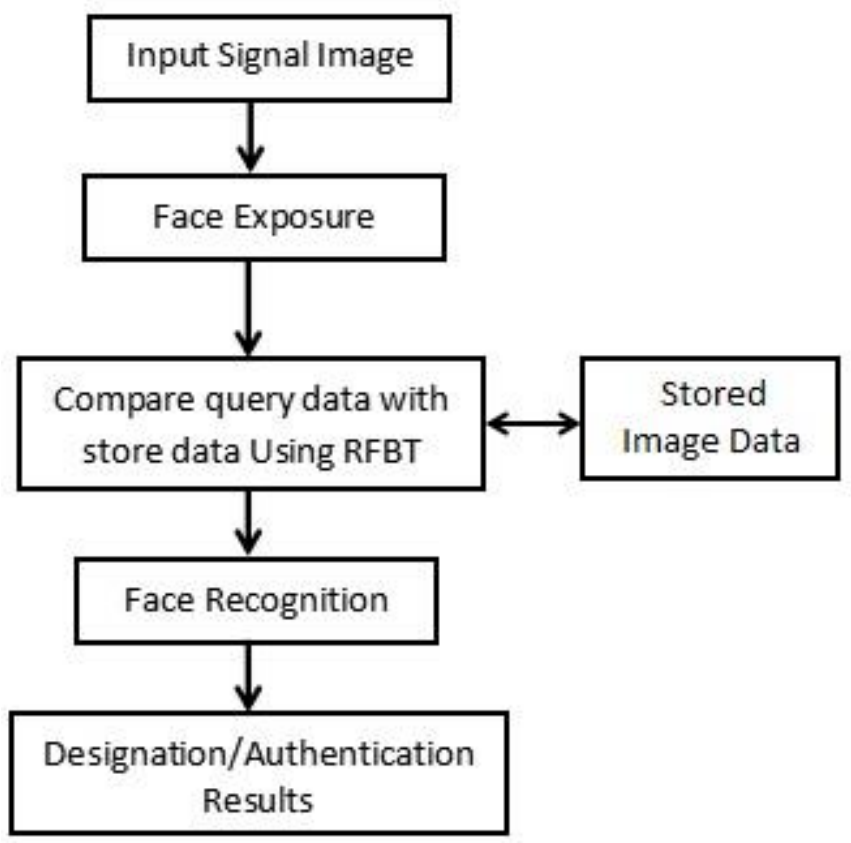

Figure.6. Essential flow chart of Unconstrained Face Recognition using RFBT

Figure-6 shows the essential flowchart of Unconstrained Face Recognition using RFG. Firstly, the input signal which consists of either image or video is taken, and then it passed 
through face exposure process which uses phrase tracking and pose judgment etc. This is further processed to extract characteristics such as look tracking impression identification. Then the data probed is graphed and compared with stored image/video of face from the data base Graph. If it matches then the unconstrained face recognition complete and this process utilizes RFG. At last authentication result will be displayed.

\section{RESULTS AND DISCUSSION}

In this research work a novel RFBT centered strategy towards face-recognition in real world situations is being suggested. The substantial scientific tests on open sources that are accessible show that the recommended techniques; will outperform their state-of-the-artwork techniques with feature types that are related. Experience will not be required by the recommended strategy positioning, which is strong to alterations in present. Outcomes on real world information with extra Problems for example size phrase, and lighting claim that the Reference face based technique strategy is also strong in these elements. The strategy that is recommended may scalable due to DCT's incorporation hashing using the detailed research encounter chart that addresses a number of experience pictures with various pose, gestures, size, and lighting.

Expected result includes the Development of face recognition techniques with enhanced performance of the proposed; reference face based technique on Effective Unconstrained Face Recognition from Image Sequence in image processing with illumination and pose variation discuss here.

Figure 7 shows the Basis Guide block diagram for RFBT. This figure shows how to create guide block for unconstrained face recognition in MATLAB and also creating the coding for development of technique.

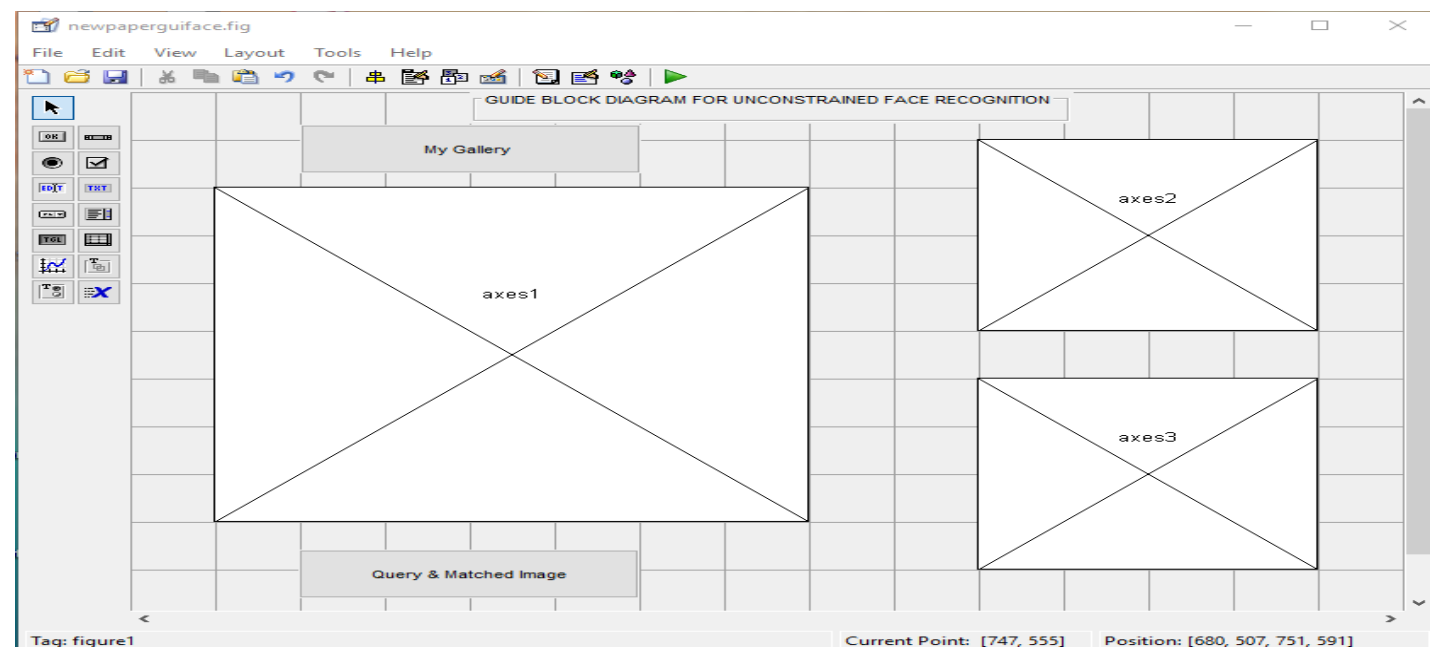

Figure.7. Basis Guide block diagram for RFBT 


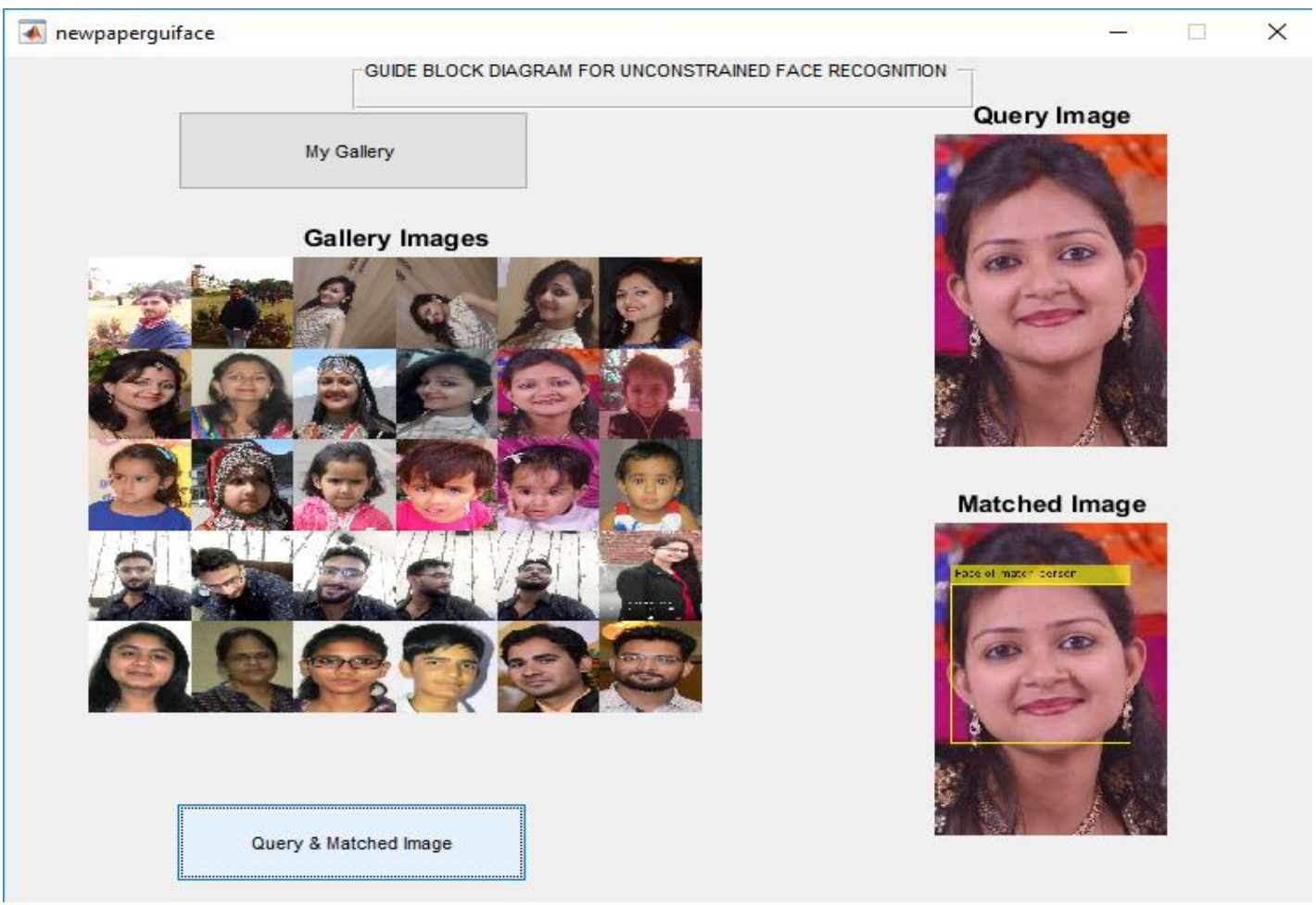

Figure.8. First sample Guide block diagram for RFBT with query and matched image for one Image.

All experiments perform on MATLAB 2017-A Version and images taken by 12 Mega pixel camera after create the data base. Figure 8 shows the First sample Guide block diagram for RFBT with query and matched image for one Image.

In figure 8, experiment one is developing. Firstly create the gallery of 30 Images and with the help of coding call the query image. This query image compare with all 30 images of gallery and after proper matching with query image to gallery images the result display on computer screen with the help of MATLAB coding.

Figure 9 shows the Second sample Guide block diagram for RFBT with query and matched image for one Image. This is experiment two of our results. It again provides the matching result with 30 images gallery and shows the exact result of matched query image. 


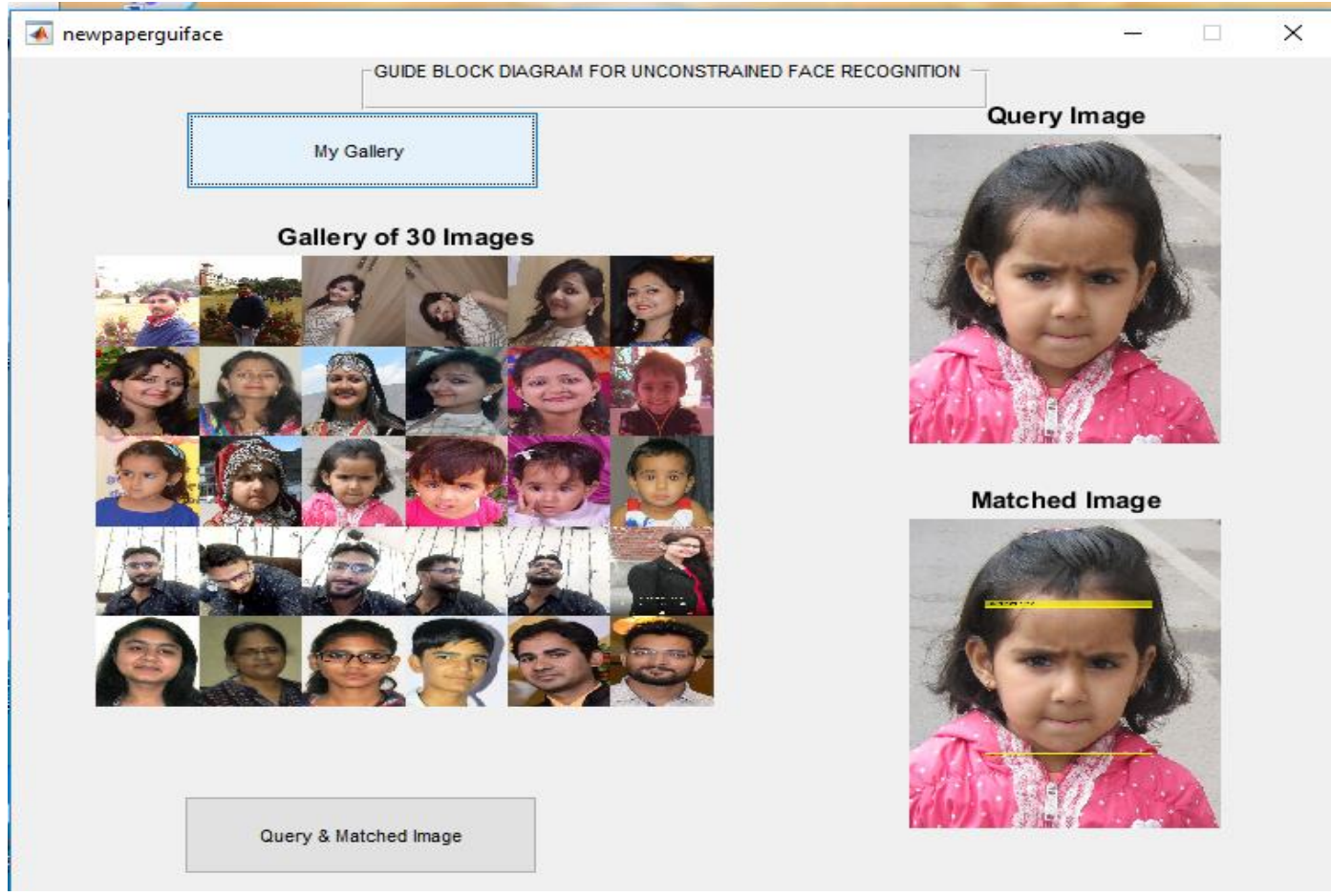

Figure .9. Second sample Guide block diagram for RFBT with query and matched image for one Image.

Figure 10 shows the Third sample Guide block diagram for RFBT with one query and two matched image from gallery images. In results Matched Image1 matched $100 \%$ with query image but Matched Image 2 is also recognized with perfect matching with different angle, position, color and environment. It shows the matching of unconstrained faces recognition using RFBT is good compare to RFG for different passions.
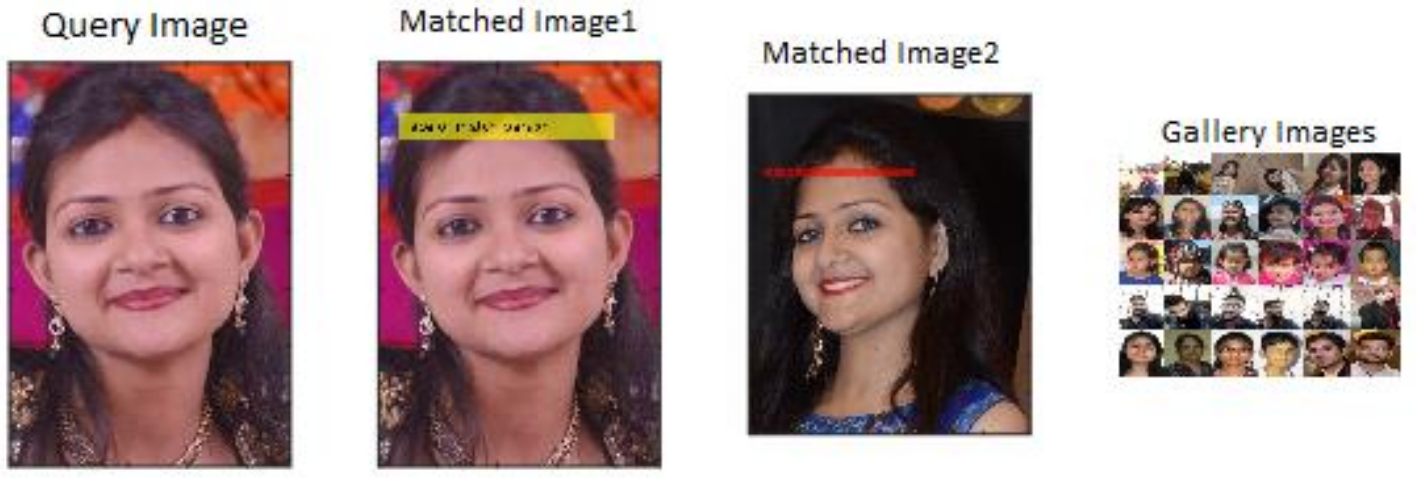

Figure.10. Third sample Guide block diagram for RFBT with one query and two matched image from image sequence. 


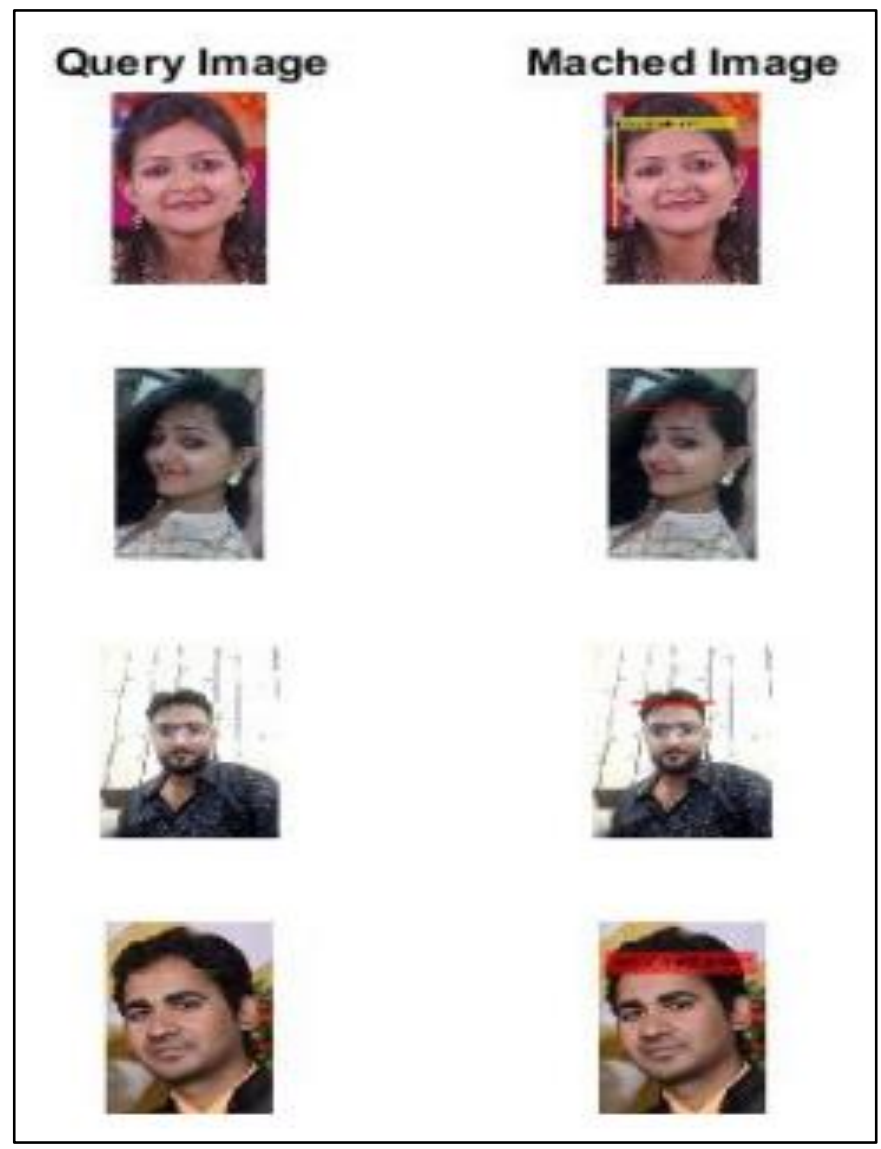

\section{Figure.11. Four query images matched with four gallery images with face recognition}

In figure 11, it shows four query images matched with four gallery images with face recognition in constrained and unconstrained environment. The results are showing perfect matching with gallery image.

\section{CONCLUSION}

A preliminary Analysis on Reference Face Based Techniques will be provided. Problems such as the common building for face recognition, factors that may affect the effectiveness of the recognizer, and several situation-of-the-art recognition techniques will be covered. In real life conditions, a distinctive RFG supported method of face recognition will be organized.

Some experience push can be utilized to raise and decide a subject in forensic face recognition. Some types of existing customization computations should boost the identification accuracy. Expected result offers the Enhancement of face-recognition techniques with improved effectiveness of the proposed, study experience chart-centered unconstrained faceacknowledgment in image processing with illumination and existing difference. This research work is totally based on algorithmic technique and simulation of improved unconstrained face recognition quality in unconstrained problems. Our method provides exact matching with reference face to gallery image in result. This work also improved for more difficulty such as angle, nose etc. parameter variation in future. 


\section{References}

[1] Ian T. Young Jan, J. Gerbrands, Lucas J. van Vliet, “Fundamental of Image Processing”, (1995).

[2] Doo Hyun Choi, Ick Hoon Jang, Mi Hye Kim, Nam Chul Kim, "Color image enhancement using single-scale retinex based on an improved image formation model", 16th European Signal Processing Conference (EUSIPCO 2008), Lausanne, Switzerland, August pp.25-29, (2008).

[3] Leila Fallah Araghi, Mohammad Reza Arvan, "An Implementation Image Edge and Feature Detection Using Neural Network" Proceedings of the International Multi Conference of Engineers and Computer Scientists, Vol I, March 18-20, (2009).

[4] Abhijith Punnappurath, Ambasamudram Narayanan Rajagopalan,Sima Taheri, Rama Chellappa, "Face Recognition Across Non-Uniform Motion Blur, Illumination, and Pose" IEEE Transactions on image processing, vol. 24, no. 7, july (2015).DOI: 10.1109/TIP.2015.2412379

[5] Rafel C. Gonzalez, Richard E. Woods, "Digital image processing", third edition, (2008). DOI: 10.1109/IEMDC.2013.6556306

[6] Mehran Kafai, Le An and Bir Bhanu "Reference Face Graph for Face Recognition", IEEE Transactions on information forensics and security, Vol.9, No.12, pp.2132-2143, 19 Sept. (2014). DOI: 10.1109/TIFS.2014.2359548

[7] Tyagi, R., Tomar, G. S., Shirvastava, "Unconstrained face recognition Quality: A review”, International Journal of Signal Processing, Image Processing and Pattern Recognition, (2016), Vol.9, No.11, pp. 199-210

[8] Tyagi, R., Tomar, G. S., Baik, N. "A Survey of Unconstrained face recognition algorithms and its application" International Journal of Security and Its Application, (2016), Vol.10, No.12, pp. 369-376

[9] "Face Recognition Applications". Animetrics. (2008)

[10] "Facial Recognition: Who's Tracking You in Public?". Consumer Reports. (2016).

[11] Jocelyn C. , Adams Kristen C. Allen , Tim Miller, Nathan D. Kalka and Anil K. Jain, "Grouper: Optimizing Crowdsourced Face Annotations" IEEE Conference on Computer Vision and Pattern Recognition Workshops (CVPRW) pp. 163-170, June (2016).DOI: 10.1109/CVPRW.2016.27

[12] Lacey Best-Rowden, Hu Han, Charles Otto, Brendan Klare, and Anil K. Jain, "Unconstrained Face Recognition: Identifying a Person of Interest from a Media Collection, IEEE Transactions on Information Forensics and Security, vol.-9, Issue-12, pp. 2144-2157, Dec.-(2014).DOI: 10.1109/TIFS.2014.2359577

[13] Tyagi, R., Tomar, G. S.: 'Tranformation of Image from Color to Gray Scale Using contrast among DPCM and LMS Method' Internation Journal of Signal Processing,Image Processing and Pattern Recognition, (2016), 9 , (8), pp. 11-24

[14] Tyagi, R., Tomar, G. S.: 'Unfamiliar Sides, video, image enhancement in face recognition' International Journal of Hybrid Information Technology, (2016), Vol.9, No.11, pp. 255-266

[15] C. Ding and D. Tao, “A Comprehensive Survey on Pose-Invariant Face Recognition” ACM Transactions on Intelligent Systems and Technology (TIST), Vol.7, No.3, pp.1-40, April (2016). DOI: 10.1145/2845089

[16] Mara Olekalns and Daniel Druckman, "With Feeling: How Emotions Shape Negotiation" in the Negotiation Journal, Volume 30, Number 6, October (2014). DOI: 10.1007/978-94-017-9963-8_2

[17] A. Punnappurath, A. N. Rajagopalan, S. Taheri, R. Chellappa, "Face Recognition Across Non-Uniform Motion Blur, Illumination, and Pose" IEEE Transactions on image processing, vol. 24, no. 7, july (2015). DOI: 10.1109/TIP.2015.2412379

[18] P. Vageeswaran, K. Mitra, and R. Chellappa, "Blur and illumination robust face recognition via set-theoretic characterization," IEEE Trans. Image Process., Vol. 22, No. 4, pp. 1362-1372, Apr. (2013).DOI: 10.1109/TIP.2012.2228498

[19] S. Sengupta, J. C. Chen, C. Castillo, V. M. Patel, R. Chellappa, and D. W. Jacobs, "Frontal to Profile Face Verification in the Wild" IEEE Winter Conference on Applications of Computer Vision (WACV), pp. 1-9, March (2016). DOI: 10.1109/WACV.2016.7477558 
[20] Jun-Cheng Chen, V. M. Patel, and R. Chellappa, "Unconstrained Face Verification using Deep CNN Features" IEEE Winter Conference on Applications of Computer Vision (WACV), pp.-1-9, March-(2016).DOI: 10.1109/WACV.2016.7477557

[21] S. Liao, Z. Lei, D. Yi, and S. Z. Li, "A Benchmark Study of Large-scale Unconstrained Face Recognition" IEEE International Joint Conference on Biometrics (IJCB), pp.1-8, sep.-29-oct.-2-(2014).DOI: 10.1109/BTAS.2014.6996301

[22] Thi Thanh Thuy Phama, Thi-Lan Lea, Hai Vua, Trung Kien Daoa and Van Toi Nguyenc, "Fully-automated person re-identification in multi-camera surveillance system with a robust kernel descriptor and effective shadow removal method" Image and Vision Computing, Publisher-Elsevier, Vol.59, pp.44-62, March (2017).DOI: 10.1016/j.imavis.2016.10.010

[23] Hardeep Kaur and Amandeep Kaur, "Illumination Invariant Face Recognition" International Journal of Computer Applications (0975 - 8887), Vol.64, No.21, pp.23-27, Feb.-(2013).

[24] C.Indhumathi, "Unconstrained Face Recognition From Blurred and Illumination with Pose Variant Face Image Using SVM” International Journal of Innovative Research in Computer and Communication Engineering (An ISO 3297: 2007 Certified Organization) Vol.2, Special Issue 1, pp. 2564-2567, March (2014).

[25] A.Devi and A.Marimuthu, "Image Processing Techniques in Face Recognition" International Journal of Computer Trends and Technology, Vol.4, No.2, pp.59-62, (2013).

[26] [Keyurkumar Patel, Hu Han and Anil K. Jain, “Secure Face Unlock: Spoof Detection on Smartphones” IEEE transactions on information forensics and security, Vol.11, No.10, October (2016). DOI: 10.1109/TIFS.2016.2578288

[27] D. Wang, C. Otto and A. K. Jain, "Face Search at Scale", IEEE Transactions on Pattern Analysis and Machine Intelligence, Vol.39, No.6, pp.1122-1136, June (2017). DOI: 10.1109/tpami.2016.2582166 
Reference Face Based Technique for Unconstrained Face Recognition from Images Gallery 